\title{
GROWTH MORPHOLOGY AND ATOMIC SURFACE TOPOLOGY BY HARTMAN-PERDOK ANALYSIS: APPLICATION TO $\mathrm{ABCO}_{4}$ AND $\mathrm{YBa}_{2} \mathrm{Cu}_{3} \mathrm{O}_{7-x}$
}

\author{
C.F. WOENSDREGT \\ Faculty of Earth Sciences, Geodynamical Research Institute \\ P.O. Box 80.021, 3508 TA Utrecht, The Netherlands
}

The Hartman-Perdok theory explains the relation between crystal structure and morphology and provides the atomic surface topology of the crystalmelt interface. Hartman-Perdok theory has been applied to $\mathrm{CaYAlO}_{4}$ as model for all other $\mathrm{ABCO}_{4}$ compounds with a $\mathrm{K}_{2} \mathrm{NiF}_{4}$ crystal structure. $\dot{F}$ forms are $\{002\},\{101\},\{103\},\{110\},\{112\},\{200\},\{211\}$ and $\{213\}$. The strongly anisotropic shape caused by the perovskite-like $\mathrm{AlO}_{6}$ layers $\|\{001\}$ is very distinct in all theoretical growth forms. The form with formal charges is planar following $\{001\}$ with $\{101\}$ and $\{110\}$ as lateral forms. Disordering of the boundary ions results in the disappearance of $\{110\}$. At lower effective charge on oxygen ions, $q_{\circ}$, the ordered forms are still tabular, while $\{110\}$ and $\{112\}$ are the only lateral faces. At still lesser negative $q_{\circ}\{112\}$ appears as well. On the disordered models $\{112\}$ replaces $\{110\}$. Crystals show often variations in colour parallel to the $\{110\}$ interface due to the surface topology of $\{110\}$. YBa2 $\mathrm{Cu}_{3} \mathrm{O}_{7-x}$ has, for $x=1$, the following $F$ forms: $\{001\}$, $\{101\},\{103\},\{112\}$ and $\{114\}$. The theoretical growth form of this tetragonal phase is tabular following $\{001\}$ with $\{101\}$ as lateral form. For $x=0$ the growth form shows important $\{101\}$ and minor $\{103\}$ and $\{001\}$. When the boundary ions on (001) are ordered, the outermost layer of $\{001\}$ contains half of the $\mathrm{Cu}^{+}(x=1)$ or $\mathrm{Cu}^{3+}$ and $\mathrm{O}^{2-}(x=0)$ ions in a $c(2 \times 2)$ quadratic lattice which reduces the $\{001\}$ growth rate significantly. An $(1 \times 2)$ reconstructed $\{010\}$ surface can be traced for the orthorhombic polymorph which results into the appearance of $\{010\}$ on the ordered growth form. Otherwise the presence of $\{010\}$ on as-grown crystals must be due to external factors.

PACS numbers: 68.35.Bs, 68.35.-p, 81.10.Aj

\section{Hartman-Perdok theory}

The relation between the internal crystal structure and the crystal morphology can be established by the Hartman-Perdok theory [1-3]. At the same time the surface topology of the crystalline interface can be derived at atomic scale.

The crystal morphology is not only determined by internal factors such as crystal structure, twinning, dislocations, etc., but also by external factors such 
as supersaturation, the presence of impurities including the solvent, temperature, pressure and environmental symmetry.

Crystal faces are crystalline interfaces during the process of crystal growth. Sometimes habit changes occur since external growth conditions such as supersaturation and/or impurity concentrations change during the growth. The surface topology of these crystalline interfaces determines surface diffusion, incorporation of the growth units into kinks or steps, adsorption, etc.

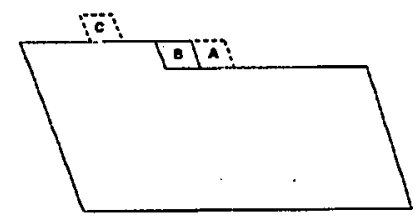

Fig. 1. When a strong bond exists between $A$ and $B$ as shown in a two-dimensional crystal, the probability of detachment of growth unit $A$ is less than that of $C$.

On a two-dimensional crystal, see Fig. 1, the crystallising units are represented by small blocks. Growth may take place by the attachment of crystallising units to the crystal surface at the kink sites such as $B$. Now the crystal will increase in size when the flux of attaching crystallising units is larger than that of the detaching units. At equilibrium conditions the amount of crystallising units equals that of the dissolving units. The probability of attachment for growth units is the same for sites such as $A$ and $C$. When there exists a strong bond between the units $A$ and $B$, the detachment of unit $C$ is energetically easier than that for $A$. Hence the growth will proceed parallel to the lattice row $[u v]$ forming straight edges on the condition that the units are bonded by strong bonds parallel to that direction.

Strong bonds are in the sense of the Hartman-Perdok theory short bonds in the first coordination sphere and formed during the crystallization. The periodic bond chain $(\mathrm{PBC})$ is an uninterrupted periodic chain of strong bonds between the crystallising units, such as ions, molecules, atoms or clusters thereof, formed during crystallization. A PBC must be stoichiometric and should not have a dipole moment perpendicular to its direction [uvw]. Only a limited number of PBCs can be distinguished in a crystal structure assuming that strong bonds are confined to the first coordination sphere.

In a projection of the three-dimensional crystal structure parallel to their vector translation (Fig. 2a) the PBCs are seen end-on as open circles. The detachment of growth units parallel to the PBCs is easier for $C$ than for $A$, when additional strong bonds are present between $A$ and $B$. These latter bonds form another PBC parallel to the crystal surface. Hence the growth of such a flat or $F$ face proceeds through a layer mechanism if two or more PBCs are parallel to its surface. In Fig. 2b strong bonds between $\dot{A}$ and $B$ are absent which results in a stepped or $S$ face as the probability of detachment for $A$ and that for $C$ are equal. Crystal faces can be classified either as $F$ faces which contain at least two PBCs in a slice of thickness $d_{h k l}$, or as an $S$ face containing only one PBC, or 


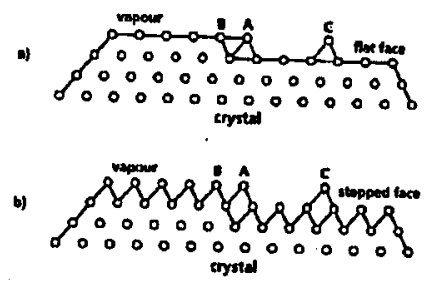

Fig. 2. Three-dimensional crystal structure has been projected parallel to the direction of the PBC. PBCs are seen end-on as open circles: (a) $F$ or flat faces grow according to a layer mechanism since they are parallel to at least two non-collinear PBCs, (b) stepped or $S$ faces are parallel to only one set of PBCs.

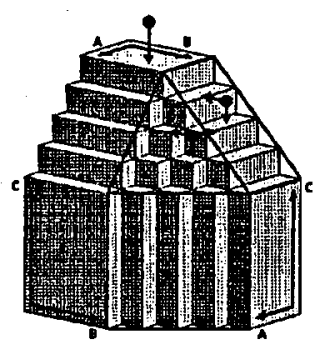

Fig. 3. Hartman cube showing classification into $F$-, $S$ - and $K$-faces as function of the presence of PBCs parallel to the $A$-, $B$ - and $C$-axes.

as $K$ faces containing no $\mathrm{PBC}$ at all. This is also schematically demonstrated in Fig. 3. The $F$ faces have the lowest growth rates as they grow according to a layer mechanism (two-dimensional nucleation or BCF spiral growth). $K$ faces are microscopically rough and grow quickly without nucleation. $S$ faces are intermediate between $F$ and $K$ faces. Although they need an one-dimensional nucleation, in practice there are always sufficient kinks present on the steps. Therefore these $S$ faces grow almost as fast as $K$ faces. This means that neither $K$ faces nor $S$ faces should be present on growth forms, which are crystals grown in nature or laboratory experiments from a supersaturated medium.

The identification of PBCs and subsequent classification of crystal faces as $F, S$ and $K$ faces can be of great value in understanding the crystal morphology. The slice with a thickness of $d_{h k l}$ and having an $F$ character defines the elementary growth layer. At the same time the slice boundary represents the surface of the crystalline interface during the crystal growth process at atomic scale. Hence the relation between the crystal structure and the atomic surface configuration of the crystalline interface can be derived by the Hartman-Perdok theory.

The specific surface energy is determined by the surface structure of a growing crystal at atomic scale. The distance between two identical surfaces of $(h k l)$ is equal to the interplanar spacing $d_{h k l}$. When the unit cell is centred and/or screw axes or glide planes are present, $d_{h k l}$ may be a submultiple, $d_{n h n k n l}$, where $n=2,3, \ldots$ Hence the same extinction rules must be applied as for X-ray reflec- 
tions. According to the Hartman-Perdok theory the slice should be considered as the elementary growth layer.

The attachment energy, $E_{\mathrm{a}}$, is the energy per mole released, when a new slice of thickness $d_{h k l}$ crystallises on an already existing crystal face $(h k l)$. The attachment energy is, at least for $F$ faces, assumed to be directly proportional to their growth rates [4]. The theoretical growth form can be constructed by taking the attachment energy, $E_{\mathrm{a}}^{h k l}$, as directly proportional to the growth rate of face $(h k l)$ in the Wulff plot.

Hence according to the Hartman-Perdok theory the external morphology can be derived from the crystal structure under the conditions that the growth rate is controlled by interface kinetics and that the dislocation density of this interface does not influence the growth rate or, otherwise said, it is assumed to be uniform for all faces. Moreover, the external factors are assumed to be the same for all faces. For the qualitative PBC analysis holds that the kinetics are determined by the formation of strong bonds during the crystallization.

\section{2. $\mathrm{ABCO}_{4}$ compounds}

\subsection{Crystal structure}

Tetragonal $\mathrm{ABCO}_{4}$ compounds, where $\mathrm{A}=\mathrm{Sr}, \mathrm{Ca} ; \mathrm{B}=$ rare-earth elements and $\mathrm{C}=\mathrm{Ga}$ or $\mathrm{Al}$, are very suitable substrates for epitaxial growth of thin films of high- $T_{c}$ superconductors. They are chemically stable and have very low dielectric constants. Moreover, their lattices match very well with those of superconductors [5-7]. The Hartman Perduls thoury has been applime to CaYAlO, which can be
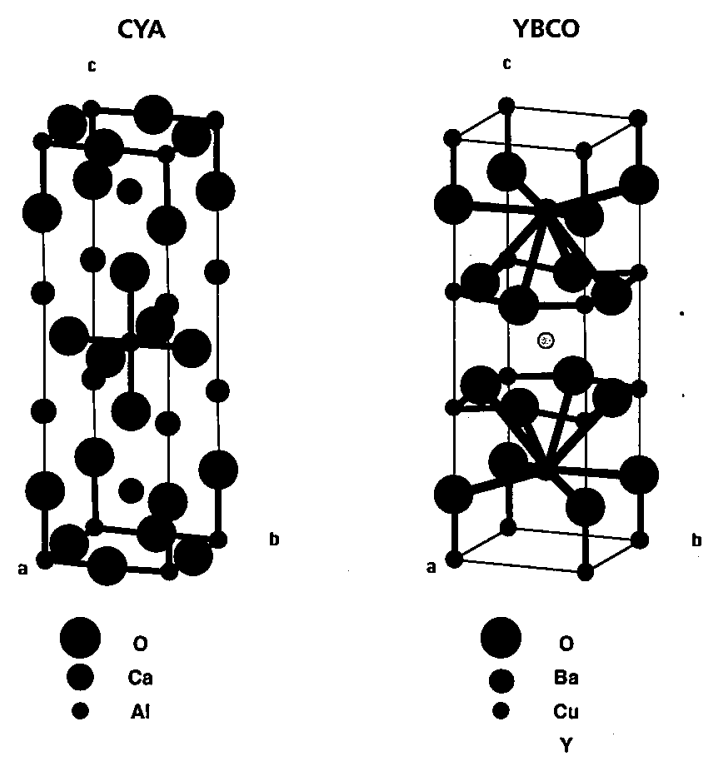

Fig. 4. Crystal structure of (a) $\mathrm{CaYAlO}_{4}$ and (b) $\mathrm{YBa}_{2} \mathrm{Cu}_{3} \mathrm{O}_{7-x}$. 


\section{TABLE}

Cell constants in $\mathrm{nm}$ and fractional atomic coordinates.

\begin{tabular}{l|c|c|c}
\hline \hline & $\begin{array}{c}\mathrm{CaYAlO}_{4} \\
\text { (CYA) [9] }\end{array}$ & $\begin{array}{c}\mathrm{YBa}_{2} \mathrm{Cu}_{3} \mathrm{O}_{7-\mathrm{x}} \\
(\mathrm{YBCO})[11]\end{array}$ \\
\hline$a_{0}$ & 0.36451 & \multicolumn{2}{c}{0.3861} \\
$c_{0}$ & 1.18743 & \multicolumn{2}{c}{1.1774} \\
Space group & $I 4 / m m m$ & \multicolumn{2}{c}{$P 4 / m m m$} \\
\hline \multicolumn{1}{c|}{ Atom } & $x$ & $y$ & $z$ \\
\hline $\mathrm{Al}(1)$ & 0.0000 & 0.0000 & 0.0000 \\
$\mathrm{Ca} / \mathrm{Y}(1)$ & 0.0000 & 0.0000 & 0.3582 \\
$\mathrm{O}(1)$ & 0.5000 & 0.0000 & 0.5000 \\
$\mathrm{O}(3)$ & 0.0000 & 0.0000 & 0.1679 \\
\hline \multicolumn{4}{|c|}{ Atomic coordinates of YBCO } \\
\hline \multicolumn{1}{c|}{ Atom } & $x$ & $y$ & $z$ \\
\hline $\mathrm{Y}(1)$ & 0.5000 & 0.5000 & 0.5000 \\
$\mathrm{Ba}(1)$ & 0.5000 & 0.5000 & 0.1938 \\
$\mathrm{Cu}(1)$ & 0.0000 & 0.0000 & 0.0000 \\
$\mathrm{Cu}(2)$ & 0.0000 & 0.0000 & 0.3617 \\
$\mathrm{O}(1)$ & 0.0000 & 0.0000 & 0.1553 \\
$\mathrm{O}(3)$ & 0.5000 & 0.0000 & 0.3786 \\
\hline
\end{tabular}

Note that in case of $\mathrm{YBa}_{2} \mathrm{Cu}_{3} \mathrm{O}_{7}$ an additional $\mathrm{O}$ atom is statistically divided over two positions: $(1 / 2,0,0)$ and $(0,1 / 2,0)$.

considered as a suitable model for all other $\mathrm{ABCO}_{4}$ compounds with a $\mathrm{K}_{2} \mathrm{NiF}_{4}$ crystal structure such as $\mathrm{SrLaGaO}_{4}$ and $\mathrm{CdNdAlO}_{4}$ [8].

These $\mathrm{ABCO}_{4}$ compounds are tetragonal with space group $I 4 / \mathrm{mmm}$ (Fig. 4a). The fractional coordinates and lattice constants of $\mathrm{CaYAlO}_{4}$ [9] are listed in Table. According to the extinction rules of this space group the elementary growth layers called slices with thickness of $d_{h k l}$ must comply with the condition that $h+k+l=2 n$. The aluminium is sixfold coordinated by oxygen (bond lengths: four times 0.18225 and twice $0.19937 \mathrm{~nm}$ ). There are no indications that the calcium and yttrium ions are ordered. They are surrounded by nine oxygen ions belonging to three different Al-O octahedra (bond lengths vary between 0.22597 and $0.25960 \mathrm{~nm}$ ). Strong bonds could be limited to the bonds between $\mathrm{Ca} / \mathrm{Y}$ and the $\mathrm{AlO}_{6}$-octahedra. These octahedra are, however, not present as isolated units but are forming layers $\|$ (001). Hence Al-O-Al bonds must be formed, at least partly, during crystallization. Slices are never defined in such a way that $\mathrm{Al}$ ions are situated on the slice boundaries. In such a situation another slice boundary configuration has been selected which cuts through a smaller number of Al-O bonds and is always energetically more favourable. 


\subsection{Determination of $F$ faces}

The search for PBCs and $F$ faces will be described for three forms belonging to the zone of the $\langle 100\rangle$ PBC. Two proto-PBCs (pPBCs) are present parallel to $\langle 100\rangle$. A pPBC is the backbone of a complete PBC and does not necessarily comply with the conditions of stoichiometry and nonpolarity. The pPBCs present parallel to this shortest translation period within the tetragonal $I$ cell (Fig. 5, lower part) are

a) $\mathrm{Al}(1)-\mathrm{O}(6)-\mathrm{Al}(1,100)$

and

b) $\mathrm{Ca} / \mathrm{Y}(4)-\mathrm{O}(3,100)-\mathrm{Ca} / \mathrm{Y}(4,100)$.

The pPBC(a) is the chain of corner sharing octahedra parallel to $\langle 100\rangle$, while the pPBC $(b)$ is part of the double zigzag chains of $\mathrm{Ca} / \mathrm{Y}-\mathrm{O}-\mathrm{Ca} / \mathrm{Y}-\ldots$ in between the layers of $\mathrm{AlO}_{6}$ octahedra.

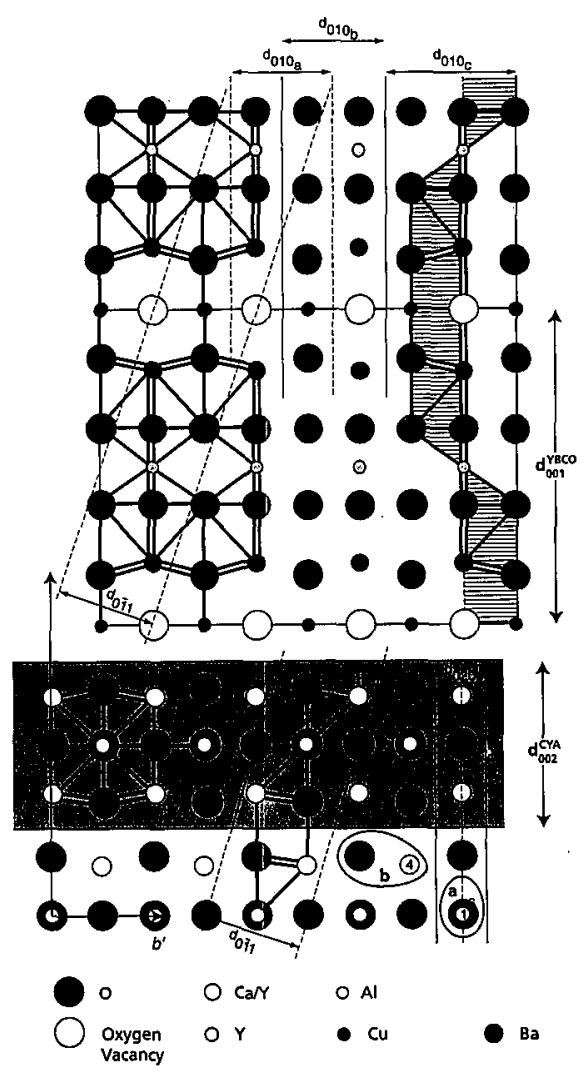

Fig. 5. Epitaxial intergrowth of $(001)_{\mathrm{YBa}_{2} \mathrm{Cu}_{3} \mathrm{O}_{7-x}}$ on top of $(001)_{\mathrm{CaYAlO}_{4}}$ visualised in a projection $\|[100]_{\mathrm{CaYAlO}_{4}}$. 
The following slices $d_{0 k l}$ can be described parallel to $\langle 100\rangle$, see also Fig. 5 , lower part.

a) Slice $d_{002}$. The strongly anisotropic slice configuration of $\{001\}$ with strongly interconnected sheets of $\mathrm{Al}$ octahedra is clearly visible. Within the slice $d_{002}$ the Al-O octahedra are connected by corner sharing $\|\langle 100\rangle$. The surface of $\{002\}$ is occupied by an alternating series of oxygen ions at the apices of the octahedra and the intercalating calcium/yttrium ions.

b) Slice $d_{011}$. Within the slice $d_{011}$ the [100] PBCs are connected by another PBC || [1/2 1/2 1/2]. This PBC has one of the shortest translation periods due to the $I$ centring of the tetragonal unit cell. The surface of $\{011\}$ is occupied by oxygen ions of the octahedra corners. These ions are widely spaced and just on the surface boundary, while an array of calcium/yttrium ions is present just beneath the surface (Fig. 6a).
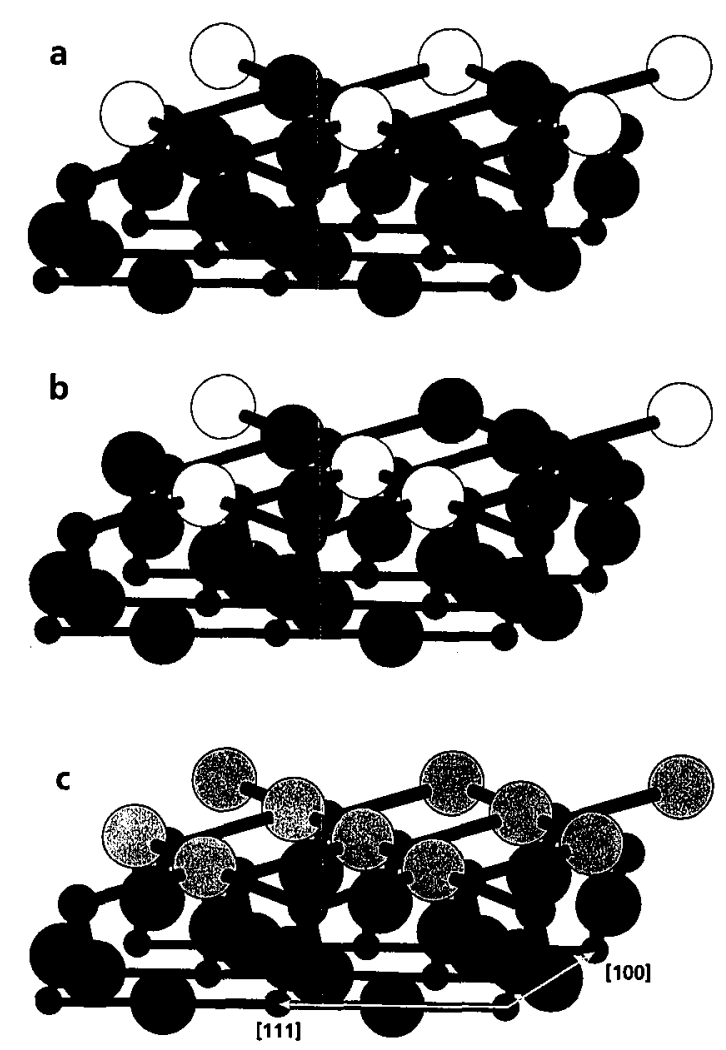

Fig. 6. Atomic surface configuration of $\{011\} \mathrm{CaYAlO}_{4}$ : (a) disordered configuration in which the light grey large spheres represent oxygen positions with an occupancy of $50 \%,(b)$ and (c) are two differently ordered surface configurations in which open large spheres indicate oxygen vacancies, dark grey spheres - oxygen ions, small grey spheres - $\mathrm{Ca} / \mathrm{Y}$ and small dark spheres - Al. 
For the boundary oxygen ions two different configurations are possible: (1) a disordered configuration, and (2) an ordered configuration. The site occupancy of the disordered boundary sites is 0.5 . Halving of the boundary ions means physically that these sites are occupied during the growth only for $50 \%$ or otherwise said that $n$ ions are statistically distributed over $2 n$ boundary sites. In the ordered configuration the same amount of ions is distributed in an ordered manner. The site occupancies are either 0.0 (for $n$ sites) or 0.5 (also for $n$ sites) as is demonstrated in Fig. 6b,c for two different configurations. The ordered configurations are always energetically more favourable, but the probability of such an ordered structure might be low at high growth rates. When such an ordering is possible the growth rate is at the minimum directly related to the $E_{\mathrm{a}}^{\text {ord }}$ and at the maximum to $E_{\mathrm{a}}^{\text {dis }}$.

c) Slice $d_{020}$. Within the slice which has a thickness of $d_{020}$ the $[100]_{\mathrm{a}} \mathrm{PBCs}$ are mutually connected by another set of PBCs parallel to [001]. For that reason $\{020\}$ must be considered as an $F$ form. The character of this $F$ face is different from that of $\{011\}$ since one more $\mathrm{Al}-\mathrm{O}$ bond is broken by the slice boundaries than in case of the previous slices.

According to the complete analysis by means of the IIartman-Perdok theory [8] the $F$ forms being parallel to at least two PBCs are in order of decreasing $d_{h k l}:\{002\},\{101\},\{103\},\{110\},\{112\},\{200\},\{211\}$ and $\{213\}$. Ordering of surface boundary ions is not only possible for $\{011\}$, but also for $\{013\},\{110\},\{211\}$ and $\{213\}$.

\subsection{Theoretical growth forms}

Attachment energies calculated in electrostatic point charge models [10] can be used for the construction of theoretical growth forms. Because the $\mathrm{Al}-\mathrm{O}$ bonds are partially covalent, $E_{\mathrm{a}}^{h k l}$ has not only been computed in models with formal charges, but also in additional models with reduced effective charges on oxygen, $q \mathrm{O}$.

The attachment energies have been computed for all $F$ faces, and in particular for $\{011\},\{013\},\{110\},\{121\}$ and $\{123\}$ both with an ordered and a disordered

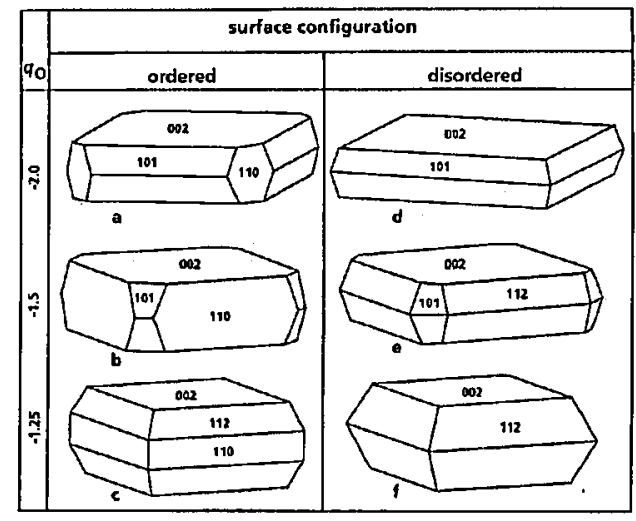

Fig. 7. Theoretical growth forms of $\mathrm{CaYAlO}_{4}$ as function of $q_{\mathrm{O}}$ for ordered and disordered surface configurations. 
boundary ion configuration. The values of $\{002\},\{112\}$ and $\{020\}$ have been used in combination with those of the ordered configurations for the construction of the ordered theoretical growth forms (Fig. 7a-c) and with the values of the disordered configurations for the disordered theoretical growth forms (Fig. $7 \mathrm{~d}-\mathrm{f}$ ).

The strongly anisotropic shape caused by the perovskite-like $\mathrm{AlO}_{6}$ layers $\|\{001\}$ is clearly visible in all models. For the formal electric charges the ordered growth form is planar following $\{001\}$ with $\{101\}$ and $\{110\}$ as lateral forms (Fig. 7a). Disordering of the boundary ions results in the disappearance of $\{110\}$ (Fig. $7 \mathrm{~d}$ ). At lower effective $q_{\mathrm{O}}$ the ordered theoretical growth forms are still tabular but the morphological importance of the lateral forms is changing. At $q_{\mathrm{O}}=-1.5|e|$ (Fig. $\left.7 \mathrm{~b}\right)\{110\}$ is mucl more important than $\{101\}$. At $q_{\mathrm{O}}$ lesser negative than $-1.4|e|$ (Fig. $7 \mathrm{c}$ ) $\{112\}$ and $\{110\}$ are the only lateral forms. For the disordered models the most striking fact is that $\{110\}$ is always replaced by $\{112\}$, see Fig. 7e, f.

\section{3. $\mathrm{YBa}_{2} \mathrm{Cu}_{3} \mathrm{O}_{7-x}(\mathrm{YBCO})$}

\subsection{Crystal structure}

Crystals of $\mathrm{YBa}_{2} \mathrm{Cu}_{3} \mathrm{O}_{7-x}$ are always grown at temperatures higher than the transition temperature of about $680^{\circ} \mathrm{C}$ below which the tetragonal crystal structure $(x=1)$ transforms into the orthorhombic phase $(x=0)$. The crystal structure data of the tetragonal pliase [11] are listed in Table. Although the lattice constants are very similar to those of $\mathrm{CaYAlO}_{4}$ (CYA), the crystal structure itself is quite different. The YBCO crystal structure for $x=0$ (Fig. $4 \mathrm{~b}$ ) can be characterised as a layer structure parallel to (001) of subsequent layers of $\mathrm{CuO}_{1-x}-\mathrm{BaO}-\mathrm{CuO}_{2}-\mathrm{Y}-\mathrm{CuO}_{2}-\mathrm{BaO}-\mathrm{CuO}_{1-x}-\ldots$ The $\mathrm{Cu}$ atoms of the $\mathrm{CuO}_{2}$ layer are fivefold coordinated (four bonds of $0.1941 \mathrm{~nm}$ and one bond of $0.2430 \mathrm{~nm}$ ). Those $\mathrm{Cu}$ atoms of the $\mathrm{CuO}_{1-x}$ layer are twofold coordinated in the tetragonal phase and fourfold in the orthorhombic phase. The central $\mathrm{Y}$ atom has an eightfold coordination (bond length of $0.2402 \mathrm{~nm}$ ) just as the Ba atoms (four times $0.2768 \mathrm{~nm}$ and four times 0.2768 ).

\subsection{Hartman-Perdok analysis}

The application of the IIartman-Perdok theory can be summarised as follows [12]. For the tetragonal crystal structure the following $F$ forms exist: $\{001\},\{101\}$, $\{103\},\{112\}$ and $\{114\}$. In the [100] projection (Fig. 5, upper part) the $F$ slices $d_{001}$ and $d_{0 \overline{1} 1}$ are outlined. The theoretical growth form of the tetragonal phase is tabular following $\{001\}$ with $\{011\}$ as lateral forms (Fig. 8a, b).

Exactly on the slice boundaries of $(001) \mathrm{Cu}^{+}$ions $(x=1)$ or $\mathrm{Cu}^{3+}$ and $\mathrm{O}^{2-}$ ions $(x=0)$, are situated. The most stable configuration is that of a centred quadratic lattice with sides $2 a$ of which the $\mathrm{Cu}^{+}$ions occupy the nodes and centre of the lattice showing presumably an ordered arrangement of an $(1 \times 2)$ structure (see for more details [12], Fig. 4). When these ordering of the $\mathrm{Cu}^{+}$ions would be possible, the growth rate of (001) will be reduced by a factor of 0.74 in case of $\mathrm{YBa}_{2} \mathrm{Cu}_{3} \mathrm{O}_{6}$. For $\mathrm{YBa}_{2} \mathrm{Cu}_{3} \mathrm{O}_{7}$ the reduction is lower (0.53). 

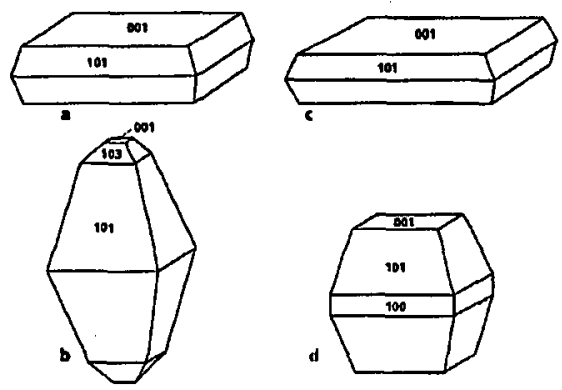

Fig. 8. (After [10].) Theoretical growth forms of $\mathrm{YBa}_{2} \mathrm{Cu}_{3} \mathrm{O}_{6}$ : (a) disordered surface boundary model, (b) ordered boundary ions $\left(\mathrm{Cu}^{+}\right.$and $\left.\mathrm{O}^{2-}\right)$ on $\{001\}$, and theoretical growth forms of $\mathrm{YBa}_{2} \mathrm{Cu}_{3} \mathrm{O}_{7}$ : (c) disordered model, (d) surface of $\{001\}$ ordered as in (b), while the surface structure of $\{010\}$ is ordered as described for $d_{010 c}$ in Fig. 5, upper part.

The crystal form $\{010\}$ is an $S$ form, because there are two different slices for $\{010\}$, i.e. $d_{010 a}$ and $d_{010 b}$ (Fig. 5, upper part). Their surface energies are exactly identical due to the presence of a symmetry centre at the origin. In that case the elementary growth layer must be halved [13]. The slice with a thickness of $d_{020}$ has an $S$ character as the [100] PBCs are not connected parallel to [u0w] within this slice. This means that the presence of $(010)$ cannot be explained when neither surface reconstruction nor adsorption of impurities or complexes would take place at this crystalline interface. For the orthorhombic $\mathrm{YBa}_{2} \mathrm{Cu}_{3} \mathrm{O}_{7}$ the slice $d_{010 c}$ (Fig. 5, upper part) is an $F$ slice as the [100] PBCs are connected parallel to [301] and [301] due to the additional oxygen atom at $(0,1 / 2,0)$. Calculations of the attachment energy indicate that $(010)$ with an ordered $(1 \times 2)$ surface structure (see Fig. 5, upper part) could be present on the theoretical growth form (Fig. 8d).

\section{Relation between $\mathrm{CaYAlO}_{4}$ substrate and $\mathrm{YBa}_{2} \mathrm{Cu}_{3} \mathrm{O}_{7-x}$}

Several $\mathrm{ABCO}_{4}$ compounds such as $\mathrm{SrLaGaO}_{4}$ (SLG) [14], $\mathrm{CaNdAlO}_{4}$ (CNA) [15] and $\mathrm{SrLaAlO}_{4}$ (SLA) [16] have already been used experimentally as substrates for $\mathrm{YBa}_{2} \mathrm{Cu}_{3} \mathrm{O}_{7-x}$ thin films. For all compounds the $\{001\}_{\mathrm{ABCO}} /(001)_{\mathrm{YBCO}}$ has been investigated, while for $\mathrm{SrLaGaO}_{4}$ [14] also $(001)_{\mathrm{SLG}} /(100)_{\mathrm{YBCO}}$ and $\{110\}_{\text {SLG }} /(110)_{\text {YBCo }}$ have been reported.

\section{1. $(001)_{\mathrm{YBCO}} /(001)_{\mathrm{CYA}}$}

The surface of the (001) CYA substrate consists of the oxygen ions located at the apices of the $\mathrm{Al}$ centred octahedra with intercalating ninefold coordinated $\mathrm{Ca} / \mathrm{Y}$ ions. Such a pattern can be recognised in the YBCO structure in the form of $\mathrm{CuO}_{2}$ layers with the fivefold coordinated $\mathrm{Cu}$ ions and the eightfold coordinated $\mathrm{Ba}$ ions. In Fig. 5 the two structures have been drawn in such a way that the best fit has been obtained between these two crystal structures for the orientation

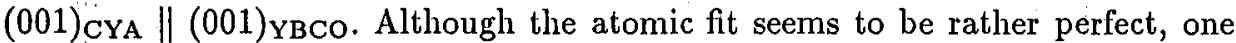
specific misfit must not be forgotten. The CYA layering consists of $\mathrm{AlO}_{6}$ octahedra 
centred at 0.0 and 0.5 height above the projection plane, while in the YBCO structure the $\mathrm{Cu}$ ions belonging to the $\mathrm{CuO}_{2}$ and $\mathrm{CuO}_{1-x}$ layers are always at the same height (0.0).

\section{Discussion and conclusions}

These studies on the theoretical crystal morphology of $\mathrm{ABCO}_{4}$ and $\mathrm{YBa}_{2} \mathrm{Cu}_{3} \mathrm{O}_{7-x}$ (see for more details [8] and [12], respectively) describe the relation between crystal structure and the crystal growth of $\mathrm{ABCO}_{4}$ and $\mathrm{YBCO}$ crystals.

Moreover, the influence of the crystal structure on the shape of the meltcrystal interface during the Czochralski method of $\mathrm{ABCO}_{4}$ crystals is now known. $\mathrm{CdNdAlO}_{4}, \mathrm{SrLaAlO}_{4}$ and $\mathrm{SrLaGaO}_{4}$ have been grown by the Czochralski technique. At the convex crystal-melt interface facets of $\{101\}$ occur, while the cylindrical part shows $\{001\}$ and depending on the growth direction $\{100\}(\|\langle 100\rangle)$ or $\{110\}(\|\langle 110\rangle)[17,18]$. These crystal forms are also the most important $F$ faces derived by the IIartman-Perdok theory. The results of the IIartman-Perdok analysis for the morphology of Czochralski grown crystals have been critically reviewed by Pajaczkowska et al. [19].

The surface topography at atomic scale derived by the Hartman-Perdok explains growth phenomena and crystal characteristics: (a) differences in colour $[20,21]$ parallel to the $\{110\}$ interface can be the surface boundaries of $\{110\}$ on which the oxygen ions are just the most important boundary ions, (b) the strongly anisotropic character of the $\{001\}$ slice causes the cracking parallel to $\{001\}$.

The Hartman-Perdok theory provides at the same time the information at atomic scale about the structure compatibility between substrate and high- $T_{\mathrm{c}}$ (YBCO) thin films. This approach is superior to the selection procedure of a suitable substrate based on lattice matching. When thin layers grown on a substrate are considered, elementary growth layers can easily be defined. This fact has been forgotten by Guo et al. [22] during the application of their interatomic structure matcling method.

This is Geodynamics Research Institute (Utrecht University) contribution 97.

\section{References}

[1] P. Hartman, in: Crystal Growth: an Introduction, Ed. P. Hartman, North-Holland, Amsterdam 1973, Ch. 14, p. 367.

[2] P. Hartman, in: Morphology of Crystals, Ed. I. Sunagawa, Terra Scientific, Tokyo and Reidel, Dordrecht 1988, part A, Ch. 4, p. 269.

[3] C.F. Woensdregt, Faraday Discuss. 1903, 95 (1993).

[4] P. Hartman, P. Bennema, J. Cryst. Growth 49, 145 (1980).

[5] M. Berkowski, J. Fink-Finowicki, A. Pajączkowska, K. Mazur, J. Sass, in: Progress of High Temperature Superconductivity, Vol. 24, Eds. W. Gorzkowski, M. Gutowski, A. Reich, H. Szymczak, World Scientific, Singapore 1990, p. 710.

[6] R. Sobolewski, P. Gierlowski, W. Kula, S. Zarembinski, S.J. Lewandowski, M. Berkowski, A. Pajączkowska, B.P. Gorshunov, D.B. Lyudmirski, O.I. Sirotinski, IEEE Trans. Magn. 27, 876 (1991).

[7] A. Pajączkowska, SPIE-Int. Soc. Opt. Eng. 2373, 273 (1995). 
[8] C.F. Woensdregt, H.W.M. Janssen, A. Gloubokov, A. Pajączkowska, J. Cryst. Growth 171, 392 (1997).

[9] R.D. Shannon, R.A. Oswald, J.B. Parise, B.H.T. Chai, P. Byszewski, A. Pajączkowska, R. Sobolewski, J. Solid State Chem. 98, 90 (1992).

[10] C.F. Woensdregt, Phys. Chem. Miner. 19, 52 (1992).

[11] S. Katano, S. Funahashi, T. Hatano, A. Matsushita, K. Nakamura, T. Matsumoto, K. Ogawa, Jpn. J. Appl. Phys. 26, 1049 (1987).

[12] B.N. Sun, P. Hartman, C.F. Woensdregt, H. Schmid, J. Cryst. Growth 100, 605 (1990).

[13] P. Hartman, W.M.M. Heijnen, J. Cryst. Growth 63, 261 (1983).

[14] S. Hontsu, J. Ishii, T. Kawai, S. Kawai, Appl. Phys. Lett. 59, 2886 (1991).

[15] C. Tomé-Rosa, G. Jakob, M. Maul, A. Walkenhorst, M. Schmitt, P. Wagner, P. Przyslupski, H. Adrian, Physica C 171, 231 (1990).

[16] R. Brown, V. Pendrick, D. Kalokitis, B.H.T. Chai, Appl. Phys. Lett. 57, 1351 (1990).

[17] A. Pajączkowska, P. Byszewski, J. Cryst. Growth 128, 694 (1993).

[18] K. Mazur, J. Sass, A. Pajączkowska, SPIE-Int. Soc. Opt. Eng. 1845, 103 (1992).

[19] A. Pajączkowska, A. Gloubokov, A. Klos, C.F. Woensdregt, J. Cryst. Growth 171, 387 (1997).

[20] A. Gloubokov, R. Jablonski, W. Ryba-Romanowski, J. Sass, A. Pajączkowska, R. Uecker, P. Reiche, J. Cryst. Growth 147, 123 (1995).

[21] A. Pajączkowska, J. Domagala, A. Gloubokov, R. Jablonski, A. Klos, to be published in Cryst. Res. Technol.

[22] R. Guo, A.S. Bhalla, L.E. Cross, R. Roy, J. Mater. Res. 9, 1664 (1994). 